\title{
Hyperspectral Imaging of Surface-Plasmon-Enhanced Local Electric Fields by EELS with Tunable <60meV Energy Resolution
}

\author{
Patricia Abellan ${ }^{1}$, Patrick Z. El-Khoury ${ }^{2}$, Fredrik S. Hage, ${ }^{1}$ Josh Cottom, ${ }^{3}$ Alan G. Joly, ${ }^{2}$ Wayne P. \\ Hess, ${ }^{2}$ Rik Brydson ${ }^{3}$ and Quentin M. Ramasse ${ }^{1}$ \\ 1. SuperSTEM Laboratory, SciTech Daresbury Campus, Daresbury, U.K. \\ 2. Physical Sciences Division, Pacific Northwest National Laboratory, Richland, U.S.A. \\ ${ }^{3 .}$ Institute for Mater. Research, School of Chem. and Process Eng., University of Leeds, Leeds, U.K.
}

Small variations in the geometry and chemical environment of metal nanostructures can cause dramatic changes in their localized surface plasmon (LSP) resonances. An ideal characterization tool of these functional materials would thus provide structural, chemical and morphological information with subnanometer resolution, all of which can be directly correlated to their plasmonic response. Using spherical-aberration-corrected microscopes it is now possible to form bright electron probes below 1 angstrom in size. Using low-loss electron energy loss spectroscopy (EELS), the spatial distribution of the plasmon-induced electric fields can be mapped using hyperspectral imaging [1]. As an example, figure 1 shows Ag particles with different shapes and different numbers of crystalline domains (A and B), along with spatially-resolved maps of the LSP modes for similar structures (D and E) for the energy ranges indicated in the figure. Both shape and crystallinity appear to affect the plasmonic response.

By taking advantage of recent improvements in energy resolution enabled by the use of electron energy monochromators, EELS has recently been proposed as a quantitative tool to provide information on damping effects in single particles and electron kinetics of single plasmon modes [2]. Crucial to the prospect of quantitative analysis of LSPs is that the experimental energy resolution must be better than the natural line-width of the plasmon resonances, whilst retaining high enough signal-to-noise ratio to enable an accurate determination of the properties of interest [2]. The energy resolution of EELS is customarily determined by the full-width at half-maximum (FWHM) of the zero-loss (ZL) peak. The plasmon resonances, lying in the low-loss regime, often overlap with the broad tail of the ZL peak, blurring many spectral signatures of interest. Until now, several processing techniques had to be applied to overcome these issues, relying for instance on deconvolution algorithms [3] which can introduce artifacts [4]. A new generation of electron monochromators now allows for high signal-to-noise ratios while varying the energy resolution controllably, down to the $10 \mathrm{meV}$ regime [5], thus removing in many cases the need for such processing steps.

Here we present recent results aimed at spatially and spectrally resolving the plasmon resonances of individual plasmonic nanostructures and functional plasmonic devices using a Cs-corrected and monochromated Nion UltraSTEM 100MC ('Hermes') microscope with a nominal energy resolution of $10 \mathrm{meV}$. We show how the energy resolution, which also affects the attainable signal-to-noise ratio and dictates the required integration (exposure) time, can be conveniently set and tuned, depending on the inherent properties of the system of interest. Figure 1C shows as-recorded EEL spectra taken from the center of a Ag nanowire (inset) and exhibiting a narrow bulk plasmon resonance at $3.85 \mathrm{eV}$. We note that the FWHM of the peak does not decrease with decreasing energy resolution from $40 \mathrm{meV}$ to $16 \mathrm{meV}$, meaning that $40 \mathrm{meV}$ must be below the natural line width of the resonance. In this context, we will discuss the prospect of not only characterizing bare metallic nanostructures, but also interrogating chemically-functionalized plasmonic nanostructures using EELS. We note that the accessible energy 
ranges (sub-40meV) also allows us to probe molecules adsorbed onto metal nanostructures. [6]

\section{References:}

[1] J. Nelayah et al., Nature Physics, 2007, 3, 348-353.

[2] M. Bosman et al., Scientific reports, 2013, 3.

[3] R. F. Egerton in "Electron Energy-Loss Spectroscopy in the Electron Microscope", Springer (New York), 3rd Ed., 2011.

[4] E. P. Bellido et al., Microsc. Microanal., 2014, 20, 767; V. Keast and M. Bosman, Microscopy research and technique, 2007, 70, 211; S. Lazar et al., Ultramicroscopy, 2006, 106, 1091.

[5] O. L. Krivanek et al., Nature, 2014, 514, 209-212

[6] SuperSTEM is the UK EPSRC National Facility for Aberration-Corrected STEM, supported by the Engineering and Physical Science Research Council. PZE acknowledges support from the Laboratory Directed Research and Development Program at Pacific Northwest National Laboratory. WPH is supported by the US Department of Energy, Office of Science, Office of Basic Energy Sciences, Division of Chemical Sciences, Geosciences \& Biosciences.
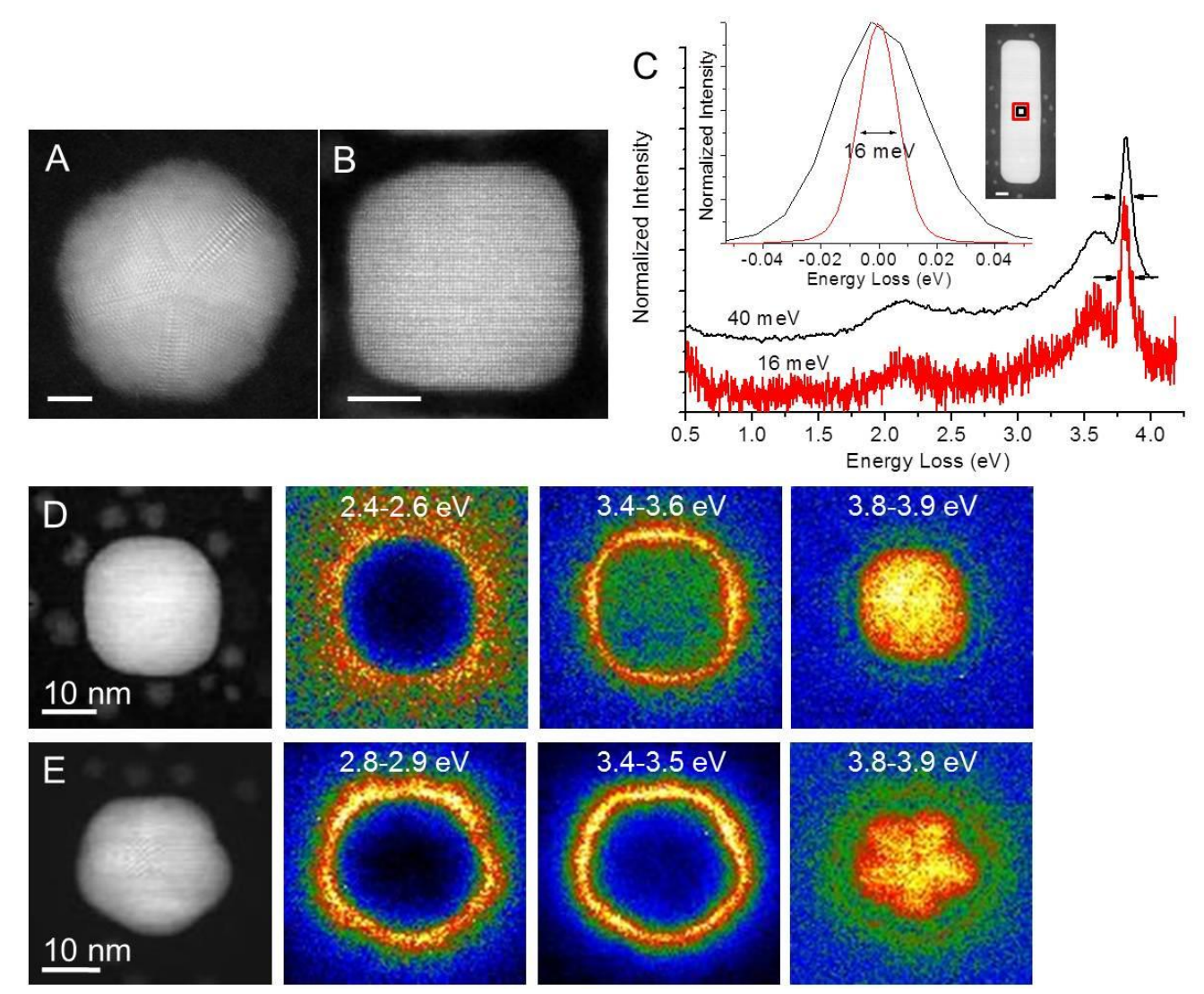

Figure 1. A) Multiply-twinned Ag particle displaying 5 rounded corners and sides. B) Monocrystalline square Ag particle with round edges. C) Example of a narrow feature unaffected by the increase of energy resolution and impact on signal-to-noise ratio. D) Spatially-resolved diagonal (left), transverse (right) LSPR modes and bulk mode of a monocrystalline Ag crystal similar to B). E) LSPR modes at corners and edges and bulk resonance for a multiply-twinned particle, similar to A). The induced electric field around the latter appears to be affected by the presence of grain boundaries. Scale bar for A), B) and inset in C) is $10 \mathrm{~nm}$. 